\title{
Bifurcation in the evolution of certainty in a small decision-making group by consensus
}

\author{
Alexandra Gheondea-Eladi ${ }^{\text {* }}$ (D) and Aurelian Gheondea ${ }^{2,3}$ \\ 'Research Institute for Quality of Life, Romanian Academy, Bucharest, Romania \\ ${ }^{2}$ Department of Mathematics, Bilkent University, Ankara, Turkey \\ ${ }^{3}$ Simion Stoilow Institute of Mathematics of the Romanian Academy, Bucharest, \\ Romania
}

\begin{abstract}
In a previous paper, the evolution of certainty measured during a consensus-based smallgroup decision process was shown to oscillate to an equilibrium value for about twothirds of the participants in the experiment. Starting from the observation that experimental participants are split into two groups, those for whom the evolution of certainty oscillates and those for whom it does not, in this paper we perform an analysis of this bifurcation with a more accurate model and answer two main questions: what is the meaning of this bifurcation, and is this bifurcation amenable to the approximation method or numerical procedure? Firstly, we have to refine the mathematical model of the evolution of certainty to a function explicitly represented in terms of the model parameters and to verify its robustness to the variation of parameters, both analytically and by computer simulation. Then, using the previous group decision experimental data, and the model proposed in this paper, we run the curve-fitting software on the experimental data. We also review a series of interpretations of the bifurcated behaviour. We obtain a refined mathematical model and show that the empirical results are not skewed by the initial conditions, when the proposed model is used. Thus, we reveal the analytical and empirical existence of the observed bifurcation. We then propose that sensitivity to the absolute value of certainty and to its rate of change are considered as potential interpretations of this split in behaviour, along with certainty/uncertainty orientation, uncertainty interpretation, and uncertainty/certainty-related intuition and affect.
\end{abstract}

\section{Introduction}

In previous research, the evolution of certainty (EoC) measured during a small-group decision process aimed at reaching consensus was shown to oscillate to an equilibrium value (Gheondea-Eladi, 2016) for about two-thirds of the experimental participants. More precisely, it was observed that, in that model, experimental participants are split into two groups, those for whom the EoC oscillates and those for whom it does not, so that a bifurcation exists. However, the mathematical model used to analyse the EoC did not explicitly embody parameters that could be related to some reasonable explanation, in psychological terms, of the observed bifurcation. For example, the bifurcation leading

*Correspondence should be addressed to Alexandra Gheondea-Eladi, Institutul de Cercetare a Calităţii Vieţii, Academia Română, Str. 13 Septembrie 1918, nr. 13, room 2.345, 050711 Bucureşti, România (email: a.gheondea.eladi@gmail.com). 
either to oscillation or evolution to equilibrium is known to be given by a number of psychological characteristics of each individual, such as certainty and uncertainty orientation (Shuper, Sorrentino, Otsubo, Hodson, \& Walker, 2004; Sorrentino, Smithson, Hodson, Roney, \& Walker, 2003), affect and intuition (Betsch \& Glöckner, 2010), and the interpretation of certainty as danger or opportunity (Kang, 2009).

In addition, mathematical models are used to analyse experimental data by means of numerical approximations, hence bifurcation phenomena may be related to subtle singularities that influence the outcome. In this respect, a refined mathematical model should be proven to be robust, that is, stable under small perturbations, in order to be a reliable tool to analyse bifurcations.

In this paper we aim to understand this bifurcation during the same consensus-based decision groups as before, to analytically emphasize the bifurcation with a new, more accurate mathematical model, which can be used to answer two main questions. First, what are the individual characteristics that lead to this bifurcation? Second, is this bifurcation amenable to the approximation method or numerical procedure?

From a psychosociological point of view, a bifurcation indicates the point at which a seemingly determined behaviour may lead to two different outcomes. In mathematics, bifurcation is a concept from the theory of dynamical systems (DSs) whereby a small change made to the parameter values of a system may cause a sudden qualitative change in its behaviour. We refine the mathematical model of the EoC to a function explicitly represented in terms of the model parameters, verify its robustness to the variation of parameters, both analytically and by computer simulation, and test the mathematical model on the experimental data. Two different EoC patterns appear as a consequence of a combination of two variables connected to individuals' sensitivity to the absolute value of certainty and to its rate of change.

The theory proposed by Zamfir (2005) states that certainty in group decisions oscillates before reaching its final value. Later, an empirical analysis of group decisionmaking aimed at reaching consensus showed that the EoC during group decisions is in fact an oscillatory dynamic process which evolves towards an equilibrium (Gheondea-Eladi, 2016). Moreover, when group members are able to report changes in their certainty for each piece of information (i.e., when communication is one-dimensional), the equilibrium value either remains constant or increases with respect to its initial value. This confirms previous research which showed that uncertainty increases during group discussions (Oskamp, 1982) and explains some cases of over- and underconfidence (Giardini, Coricelli, Joffily, \& Sirigu, 2008; Punchochar \& Fox, 2004; Slevin, Boone, Russo, \& Allen, 1998; Unal, Keating, Chytka, \& Conway, 2005) as measurements of certainty before the equilibrium value has been reached.

Although a significant part of the data analysed in previous research reflected an oscillatory character with evolution to equilibrium, some reflected more the first-order model employed (tendency to equilibrium), while others were closer to the second-order model (with oscillatory character) employed to construct the general EoC. Consequently, in this paper we look at the variables which lead to this split in the EoC in group decisions, and argue that it is given by individual sensitivity to the absolute level of the certainty and to its rate of change. By refining the previously proposed model and testing it against the experimental data as in Gheondea-Eladi (2016), it is possible to argue that the EoC for individuals who are more sensitive to how high the certainty is (sensitivity to the absolute level of the certainty) will be visibly oscillating towards equilibrium, while for those who are more sensitive to its rate of change, the oscillation to equilibrium will be less visible. 
The model-fitting results are presented in a format that is probably unusual for the social sciences and, for this reason, some observations are in order at the outset. On the one hand, our approach is rather different than the usual models, mainly due to the fact that we have a DS mathematical model in terms of differential equations in continuous time that is obtained through a mathematically rigorous procedure more common to natural sciences than to social sciences. We use mathematical modelling as a process which formulates initial hypotheses (assumptions) derived from the phenomenon to be modelled and transfers them into a formal mathematical language such that not only the interpretation of all variables is theoretically justified (based on psychological and social theories) but also that of all parameters (coefficients) of the model. Thus, choosing a mathematical modelling process as opposed to a statistical one leads to a deeper understanding of the EoC.

The DS model that we employ in this research relies on initial value problems associated to ordinary differential equations (ODEs) and, among other things, has the advantage that allows simulations that emphasize the bifurcation and an analysis of the stability to numerical perturbations. Another important advantage of the DS model is that the general model can be obtained through a subtle aggregation procedure of the two types of certainty: the objective certainty, which corresponds to a differential equation of order 1; and the subjective certainty, which corresponds to a differential equation of order 2. The resulting general model thus corresponds to a differential equation of order 3 that encompasses both the EoC and the possible oscillatory behaviour. In the absence of the modelling process employed here, only a numerical solution of the third-order differential equation would have been possible, and this would be less useful in understanding the EoC.

Our approach is based on a DS model, and there are a number of reasons for this. First, this model has the capacity to capture the oscillations of the EoC, especially in the case of subjective certainty. The eight parameters that we use in the model have clear interpretations right from the beginning, and all the mathematical and numerical operations preserve these parameters in an explicit fashion. It is this trait that enables us to connect the oscillatory or non-oscillatory behaviour to the balance between sensitivity to the level of certainty and the sensitivity to the rate of change of certainty. Second, the DS model has the potential to encode a further analysis on the overconfidence (Dunning, Griffin, Milojkovic, \& Ross, 1990; Giardini et al., 2008; Oskamp, 1982; Plous, 1995) that might show up in the EoC in decision-making groups by consensus. This can be done explicitly, by introducing more parameters which model overconfidence in subsequent research. And third, the DS model can be matched with existing models for decisionmaking in social networks which use systems of differential equations (Estrada \& VargasEstrada, 2013). In this way, our model based on a single differential equation has the potential to be adapted in future studies of the EoC in social networks.

Dynamical systems are mathematical models which were first applied in science and technology studies (Lynch, 2004; Pham, Vaidyanathan, Volos, \& Kapitaniak, 2018; Sadovnichiy \& Zgurovsky, 1986) and economics (Medio \& Lines, 2001). Applications to social psychology emerged in the 1980s and 1990s in fields such as perception, attention and human development (Vallacher \& Nowak, 1997) and have been extended to areas such as personality (Vallacher, Read, \& Nowak, 2002), women's sexual desire (Diamond, 1986, 2007), depression (Hosenfeld et al., 2015) and education (Koopmans \& Stamovlasis, 2016). Social level developments have been made in social opinion studies (Deffuant, Neau, Amblard, \& Weisbuch, 2000; Friedkin \& Johnsen, 1990), social networks (Estrada \& Vargas-Estrada, 2013) and voting behaviour (Jiao \& Li, 2021). Further DS 

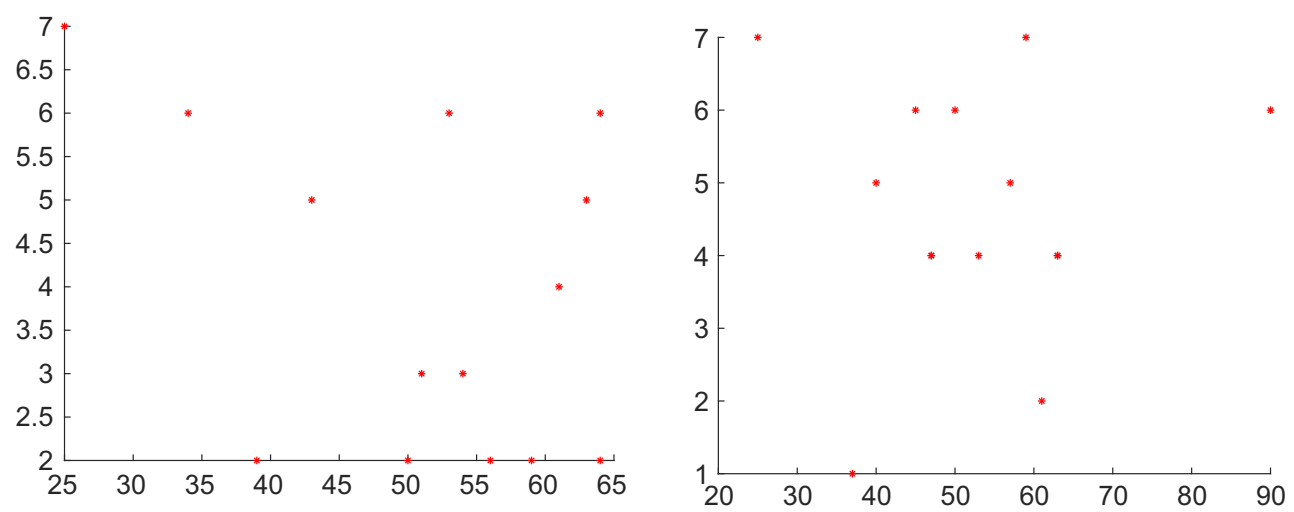

Figure 1. Scattered data for participants I1 and F1: the horizontal axis is time and the vertical axis is the level of certainty, on a scale from 1 to 7.

approaches can be linked to interval-valued fuzzy preference relations models, for example through the de Groot social network model (Shuping, Wang, \& Dong, 2018; Zhang, Zhao, et al., 2020).

Several papers provide support for social psychology researchers in the use of DS models. Carver (1997) discusses some cases of DS data interpretation. Vallacher and Nowak (1997) provide a list of methodological characteristics of linear and nonlinear dynamic models which make them suitable for social psychology: measuring intrinsic dynamic variables, phase spaces, nonlinearity, attractors, bifurcations and so on. In their response to Vallacher and Nowak's paper, Beek, Verschoor, and Kelso (1997, p. 101) point out that the search for order parameters may be deterred if no candidate variables are readily apparent. The current paper provides an example of how this may be done by considering the meaning of the parameters as it emerges from both the model and the empirical results.

Furthermore, in order to more precisely present the phenomenon of interest, in Figure 1 we plot the experimental measurements for the EoC based on the responses of two participants. These show the difficulty of finding a mathematical model simply from the scattered raw data.

Section 2 presents a review of theories of certainty and uncertainty in group decisionmaking aimed at reviewing potential interpretations of the bifurcation, as well as placing the new model within a wider theoretical perspective. After a summary of the previous model in Section 3, we present the bifurcated model of the EoC. For the convenience of the reader, we include the mathematical technicalities of obtaining the second-order model, which is responsible for the oscillation in the evolution of subjective certainty, in an Appendix, although it is a core part of the paper. In Section 4 we perform a data analysis, first by recalling the experiment, data collection and measurement, and then presenting the parameters obtained by curve-fitting on the experimental data, and explaining the numerical procedure.

\section{Literature review}

The main purpose of the literature review is to provide an interpretation of the mathematical results which will be presented in Section 3. For this purpose we review 
two bodies of literature: first, the theories of uncertainty which will later be used to interpret the main variables involved in the model; and second, the theories on consensus building which allow us to understand the influence of consensus-building practices on certainty. However, since none of the existing theories take into consideration the way in which certainty evolves during decision-making, the review in this section will look for the perspectives on certainty and uncertainty in general.

Certainty is an important aspect of any decision-making process. In social psychology, certainty and confidence are sometimes used to denote how sure a person is that a decision is correct. In other contexts, uncertainty is seen as 'the inability to determine the meaning of ... events, ... to assign definite values to objects and events and/or ... to accurately predict outcomes because sufficient cues are lacking' (Mishel, 1990, p. 256). Uncertainty is usually a state which is believed to produce discomfort, although too much certainty can lead to boredom (Huber, Sorrentino, Davidson, Eppler, \& Roth, 1992). Some researchers believe that, when measured directly, uncertainty is neither desired nor avoided, but neutral until it is appraised as either danger or opportunity (Kang, 2009; Mishel, 1990). Others have shown that when measured as a latent variable, uncertainty orientation (i.e., the desire to maintain certainty or to resolve uncertainty) leads to differences in judgements on educational and personal appraisal tasks (Sorrentino, Bobocel, Gitta, Olson, \& Hewitt, 1988), on close relationships (Sorrentino, Holmes, Hanna, \& Sharp, 1995), in health decisions (Sorrentino \& Brouwers, 1993) and in criminal conviction decisions (Hodson \& Sorrentino, 1997).

In the decision-making literature there are two main classical perspectives based on which the EoC can be analysed: groups as information processors and individuals as information processors. Each of these is further infused with two other psychological theories: the theory of motivated action and the theory of intuitive decision-making. In this section we analyse the implications of several perspectives which may inform a bifurcation theory of the EoC in group decision-making by consensus (Table 1).

In their insightful review, Hinsz, Tindale, and Vollrath (1997) noticed that groups' information processing is influenced by attention, processing space, feedback loops as well as the objectives of the information processing. Group information processing entails different structuring techniques which deal with uncertainty by enacting certain sequences in processing information which differ from group to group, task type and so on (Hirokawa \& Poole, 1996; Poole, 1983). In order to work as a unit, groups require a process of convergence of cognitive images and information necessary to make a decision (Bonito, Ervin, \& Staggs, 2016; Staggs, Bonito, \& Ervin, 2018; Zamfir, 2005).

On the other hand, De Dreu, Nijstad, and van Knippenberg (2008) suggest that motivation is needed alongside convergence and processing sequences for groups to decide under uncertainty. They argue that groups which tend to process information more deeply and thoroughly can be denoted as having high epistemic motivation. In opposition, when groups are motivated by social aspects, they are more interested in processing goal-specific information than information which is not goal-related (De Dreu et al., 2008).

When groups are regarded as sets of interacting individuals the main informationprocessing task is assumed to be performed by group members. From this point of view, individuals make decisions based on opinion samples and information collected or received from other group members (Hertwig, 2012; Rinne \& Mazzocco, 2013). However, in this process, which may be viewed as sampling of uncertain information, individuals may make judgements based on heuristics (Gigerenzer \& Brighton, 2009; Gigerenzer \& Gaissmaier, 2011; Kahneman, Slovic, \& Tversky, 1982), delegation of responsibility 
Table 1. Perspectives and theories of information processing in groups

\begin{tabular}{|c|c|c|}
\hline Level of analysis & Perspective & Sources \\
\hline Group & As information processors & $\begin{array}{l}\text { Hirokawa and Poole (1996); } \\
\text { Poole (1983) }\end{array}$ \\
\hline Group & As motivated information processors & De Dreu et al. (2008) \\
\hline Individual & $\begin{array}{l}\text { Processing information from group } \\
\text { members }\end{array}$ & $\begin{array}{l}\text { Hertwig (2012); Hertwig and Pleskac } \\
\text { (2010); Larson et al. (2019); Rinne } \\
\text { and Mazzocco (2013); van den Bos } \\
\text { and Lind (2002) }\end{array}$ \\
\hline Individual & Motivated information processor & $\begin{array}{l}\text { Birch (2009); Huber et al. (1992); } \\
\text { Shuper et al. (2004); Sorrentino and } \\
\text { Brouwers (1993); Sorrentino et al. } \\
\text { (1988), Sorrentino et al. (1995); } \\
\text { Sorrentino et al. (2003); } \\
\text { Stankov et al. (2014) }\end{array}$ \\
\hline Individual & Intuitive information processor & $\begin{array}{l}\text { Betsch (2008); Betsch and } \\
\text { Glöckner (2010) }\end{array}$ \\
\hline Individual & $\begin{array}{l}\text { Provider and receptor of information } \\
\text { and uncertainty }\end{array}$ & Gheondea-Eladi (2016) \\
\hline
\end{tabular}

(Hodson \& Sorrentino, 1997; Zamfir, 2005), frequency and variation estimates (Fidler \& Kareev, 2006; Hertwig, 2012; Hertwig \& Pleskac, 2010), and fairness (van den Bos \& Lind, 2002). At the same time, within groups, advice from others may simply be discarded, depending on whether consensus has been reached or not (Larson, Tindale, \& Yoon, 2019).

Further research argues that individuals in decision-making groups should be viewed as motivated information processors. Within this theory, individuals are believed to choose between a shallow type of analysis (e.g., heuristics, fairness, shortening information processing and decision sequence) and a deeper type of analysis (e.g., searching for options, weighting). Sorrentino and his colleagues argue that, when explaining achievement, the desire to preserve certainty (certainty orientation) or the desire to resolve uncertainty (uncertainty orientation) together with motivations for success or for avoiding failure determine individuals to take on challenging tasks (Shuper et al., 2004). On the other hand, the same certainty or uncertainty orientation has been shown to influence attitude change (Sorrentino et al., 1988) and the propensity towards groupthink (Hodson \& Sorrentino, 1997). However, in explaining mathematics achievement, Stankov, Morony, and Lee (2014) showed that confidence, past mathematics achievement, mental health and social adjustment and self-belief are actually the relevant predictors.

Another perspective proposes that individuals within decision groups consciously or subconsciously choose between using their intuition and their analytical skills (Betsch \& Glöckner, 2010; Plessner, Betsch, \& Betsch, 2008). This choice is influenced by context (Eiser \& Bhavnani, 1994; Thaler, 1985, 1999) and environment (Betsch, 2008). While the use of probabilities triggers deliberative cognitive strategies, physiological needs trigger affect-based judgement (Epstein, 2008; Weber \& Lindemann, 2008). In this way, the variables which influence certainty can be interpreted in cognitive or affective terms. 
When individuals are viewed as information processors, the context of the decision or its framing may lead to more or less cooperation within groups. Eiser and Bhavnani (1994) have shown that framing the same decision in terms of personal relations, business relations or international relations changes the more frequently chosen outcome. Studies in mental accounting showed that since different kinds of profits are processed separately, choices differ when the same task is framed in terms of money or material issues (Thaler, $1985,1999)$. On the other hand, in a health task, uncertainty is more frequently perceived as danger than opportunity (Kang, 2009), although, according to the theory of uncertainty in illness theory, it becomes problematic and impactful only if it is appraised and not before (Mishel, 1990). More than this, the locus of control (internal or external) of an individual influences the perception of uncertainty as danger or opportunity (Kang, 2009).

So far, the literature review can be summarized from two points of view: first, modelling choices; and second, determinants of uncertainty and certainty. From the point of view of modelling choices, we can summaries the literature first based on its philosophy on information and groups, and second on the applicability of a continuous or discrete model within this philosophy. First, groups can be seen as information-processing units which develop their own information-processing structures, and, at the same time, as sums of individuals processing information together. Both views may see groups and individuals as acting in a motivated fashion while being vulnerable to contextualization and framing of the decision task or making unconscious decisions based on intuition and affect when triggered by different contexts and framing. The model proposed here, like that developed in Gheondea-Eladi (2016), suggests the perspective that at a very micro level, individuals in a group are at the same time receptors and proposers of information and that, in response to this exchange, they generate perceived uncertainty. Second, the applicability of a discrete or continuous model to the perspective employed in this paper leads us to consider, on the one hand, the common points of the model proposed by Sorrentino and colleagues and that proposed by Birch. Sorrentino et al. (2003) use a discrete model. In order to deeply understand the connections to this model, a discretization of our proposal would be necessary. On the other hand, Birch (2009) proposes a model of motivated action based on streams of behaviour which account for relative frequencies of [behaviour] transitions and the distributions of transition times in a stream segment. Within this perspective, if the generation (or appraisal) of uncertainty is an act, then a sequence of such acts is a stream in itself. Thus, this model allows a continuous modelling choice, such as proposed in this paper.

From the determinants point of view, the theories reviewed suggest that subjectively perceived certainty and uncertainty are influenced by the desire to preserve certainty or to resolve uncertainty, by affect and intuition, by interpreting uncertainty as danger or opportunity, by sampling effects such as group composition (sample variation) and group decision (sample-based frequency of decision), by previous choices and goal-related motivation and by whether consensus has been reached already or not. The literature on the determinants of uncertainty and certainty may point towards an interpretation of the variables leading to the bifurcation observed, if we consider two aspects: the abstraction level at which this model is given and the fact that this split in the EoC is connected to the derivatives of the certainty function. Also, objective and subjective certainty are theoretical constructs used for building the general model of the EoC and are therefore not directly measured. Taking into account that the EoC is composed of individual-level measurements, we may consider only the variables situated at the appropriate abstraction level. Consensus achievement, goal-related motivation, previous choices, group decision 
strategy and group composition are clearly at a higher abstraction level than required here. However, certainty and uncertainty orientation, affect and intuition and the interpretation of certainty as danger or opportunity are situated at the same abstraction level as this model. Also, certainty/uncertainty orientation and certainty interpretation are directly linked to our main variable, while affect and intuition may be considered only when they are linked to it. Based on this, we suggest that future research addresses the desire to preserve certainty or to resolve uncertainty, together with certainty interpretation as danger or opportunity and affect and intuition linked to uncertainty/certainty as potential interpretations of the determinants of the observed bifurcation. These concepts ought to be considered along with the direct interpretation of the model, in terms of individuals' sensitivity to the absolute level of certainty as opposed to its rate of change.

Another body of literature which may inform the current study is on the dynamics of consensus building. In this area we pursue how different ways of reaching consensus might influence participants' levels of certainty. Certainty levels in group decision-making can be influenced by many variables, such as leadership (Bixtera \& Luhmann, 2020; Chemers, 2004), social status (Bixtera \& Luhmann, 2020; Johnson \& Ewens, 1971a; de Kwaadsteniet \& van Dijk, 2010), affective style (Johnson \& Ewens, 1971b) and physiological linkage (Thorson, Dumitru, Mendes, \& West, 2019). Interestingly, some cases of leadership are prone to lead members to reach a consensus at a lower level of confidence than expected (Estrada \& Vargas-Estrada, 2013; Locke \& Anderson, 2015). This can be the case particularly when the group decision is deferred to leaders or to external methods used to aggregate the group decision, such as in some models aimed at approximating opinion aggregation for different group structures and types, or multiattribute group decision-making with hesitant fuzzy linguistic term sets (Yu, Zhang, \& Zhong, 2018; Zhang, Gao, \& Li, 2020) or bounded confidence opinion dynamics with leaders and environmental noise (Zhao, Zhang, Tang, \& Kou, 2016).

Complex interactions between different consensus-related variables can also influence participants' certainty, for example, the perceived level of agreement, mediated by participants' assumptions on group composition (Votruba \& Kwan, 2015), confederates' congruence in answers or the incongruence in answers when the source is a highcredibility group (Hoffman, Granhag, Kwong See, \& Loftus, 2001) and group polarization - that is, group decisions situated at the already preferred pole, rather than at the other extremity (Fraser, 1971; Zhu, 2014). Group members' confidence levels, expressed verbally, non-verbally or para-verbally (Locke \& Anderson, 2015) can also influence the confidence of other members. However, confidence itself acts as a social influence only for certain types of tasks and social combination models (Zarnoth \& Sniezek, 1997).

When measuring confidence, researchers rely on peoples' ability to self-monitor and accurately perceive confidence. However, lower levels of self-monitoring influence the confidence in expert messages, while high self-monitoring influences confidence in attractive source messages (Evans \& Clark, 2012). Also, the lower the self-monitoring, the higher the confidence in the case of expert sources; and the higher the self-monitoring, the lower the confidence when the source was attractive (Evans \& Clark, 2012). On the other hand, Wichman et al. (2009) show that witnessing a second source of doubt after being primed for doubt leads to less judgements consistent with doubt than witnessing a certainty induction after being primed for doubt. To speculate on an explanation for this, it may be that witnessing doubt after doubt may mimic a doubt consensus and lead to perceiving a false sense of confidence or certainty.

Since the model proposed here does not include any consensus-related variables, we can only conjecture that out of these variables, the ones that are relevant to the current 
model are more psychological in nature (e.g., affective style and physiological linkage, selfmonitoring). The rest could be considered for further developments of this model, as will be briefly shown in Section 5.2.

\section{Mathematical model}

In this section we reshape the mathematical model for the EoC proposed in GheondeaEladi (2016) such that the bifurcation is emphasized analytically. To this end, we depart from a brief summary of the previous model of certainty and proceed to obtain the general model. Thus, the first-order ODE captures the evolution to equilibrium character, while the second-order ODE captures the oscillatory behaviour, such that the final model, of third order, will encompass both. In order to aggregate the two types of certainty evolution functions, we replace the equilibrium value of the subjective certainty with the full objective certainty function. This procedure is justified also by the fact that we found no theoretical justification for the parameters of objective certainty to be the same as those of subjective certainty. In addition, objective certainty plays the role of the equilibrium value sought through the consensus-based decision-making process. For each model we provide solutions for the relevant analytical cases and emphasize the equilibrium constant. For the general model, simulations are provided to emphasize the bifurcation when the discriminant is changing sign. In the Appendix, the model is analytically proven to be robust.

\section{I. A mathematical model for objective certainty}

In this subsection we briefly review the mathematical model of objective certainty (for details, see Gheondea-Eladi, 2016). We consider a small group of people in a process of decision-making by consensus, on a single issue (problem), in which the communication flow is a continuum variable, mathematically identified with the real positive semi-axis, while the certainty level is a function of communication. In this state-space approach, all the participants and the subgroups are providers (suppliers) of information as well as receptors (buyers) of information. The certainty with respect to the decision-making problem is considered a measurable quantity reflecting a compound concept, in which the main role is played by the receptor function, $R\left(\theta_{1}\right)$, and the provider function, $P\left(\theta_{1}\right)$, where the variable $\theta_{1}$ denotes the certainty. Both $R$ and $P$ are real-valued functions of a real variable in the state space describing the DS of the decision-making process, subject to the assumptions as in Gheondea-Eladi (2016). Each participant has a double function: of providing information, reasoning, arguments and communicating certainty (Duncan, Wengrovitz, Sedlovskaya, \& Patalano, 2007; Locke \& Anderson, 2015; Zarnoth \& Sniezek, 1997), as well as receiving all these. In psychological terms, the receptor function describes a channel through which certainty is perceived and processed, while the provider function describes an output generation channel. Based on the internalized knowledge, the provider function elicits a level of certainty which may be expressed through the designated language.

The formal representations of the functions $R$ and $P$ are

$$
R\left(\theta_{1}\right)=a_{1}-b_{1} \theta_{1}, \quad P\left(\theta_{1}\right)=c_{1}+d_{1} \theta_{1},
$$

where $a_{1}, b_{1}, c_{1}, d_{1}$ are positive real numbers: $b_{1}$ and $d_{1}$ are positive because of the decreasing/increasing behaviour of $R$ and $P$, while $a_{1}$ and $c_{1}$ are positive because it is 
important to grant some non-trivial certainty at the beginning (they can be taken nonnegative as well, but information with null certainty does not seem to be a realistic premise).

The objective certainty satisfies the first-order ODE

$$
\frac{\mathrm{d} \theta_{1}}{\mathrm{~d} t}+\left(b_{1}+d_{1}\right) \theta_{1}(t)=a_{1}-c_{1},
$$

which is made into an initial value problem (IVP) by specifying the value of $\theta_{1}$ at 0 , that is $\theta_{1}(0)=\theta_{0,1}$, a given constant.

The solution to the IVP (2), under the assumption $a_{1}, b_{1}, c_{1}, d_{1}>0$, is

$$
\theta_{1}(t)=\frac{a_{1}-c_{1}}{b_{1}+d_{1}}-\exp \left(-t\left(b_{1}+d_{1}\right)\right)\left(\frac{a_{1}-c_{1}}{b_{1}+d_{1}}-\theta_{0,1}\right)
$$

and we consider the equilibrium constant

$$
\bar{\theta}=\frac{a_{1}-c_{1}}{b_{1}+d_{1}}
$$

which will be an important part in obtaining the general model.

\subsection{A mathematical model for subjective certainty}

A realistic assumption in the case of subjective certainty is that there is a delay in the reactions of the receptors and providers of information in the decision-making group, mathematically expressible by the fact that both the receptor and the provider functions vary with the certainty function $\theta$ and its derivative $\theta^{\prime}$ as well. A delay between the objective and subjective certainty can be justified by considering that generating subjective certainty requires observing a time interval during which the rate of change of certainty is visible, while the objective certainty requires no such observation and depends solely on the absolute value of the certainty. Therefore, following the assumptions explicitly stated in Gheondea-Eladi (2016), we have the following formal representations of the functions $R$ and $P$ :

$$
R\left(\theta, \theta^{\prime}\right)=a-b \theta-g \theta^{\prime}, \quad P\left(\theta, \theta^{\prime}\right)=c+d \theta+e \theta^{\prime},
$$

where $a, b, c, d, e, g$ are positive real numbers. Therefore, the lower the values of $e$ and $g$, the lower the sensitivity to the rate of change of certainty. However, only the comparison of the aggregation of $e$ and $g$ with the aggregation of $b$ and $d$ is responsible for the bifurcation. The actual magnitude of $e$ and $g$ is of no significance for the bifurcation. The delay is the reason why the sensitivity to the rate of change appears in the model. Because of this, a discussion or experimental manipulation in terms of larger or smaller delay effects is not possible at this point. Also, if there is no delay, there is no bifurcation because there is no sensitivity to the rate of change of certainty.

Consequently, as in Gheondea-Eladi (2016), the subjective certainty is modelled by the second-order linear differential equation with constant coefficients 


$$
\frac{\mathrm{d}^{2} \theta}{\mathrm{d} t^{2}}+(e+g) \frac{\mathrm{d} \theta}{\mathrm{d} t}+(b+d) \theta=a-c
$$

in the general non-homogeneous form. This second-order ODE is usually accompanied by the initial conditions

$$
\theta(0)=\theta_{0}, \quad \theta^{\prime}(0)=\theta_{0}^{\prime}
$$

and (6) and (7) together constitute the IVP.

In order to solve the IVP as in (6) and (7), we first introduce the notation

$$
\alpha=\frac{e+g}{2}, \quad \beta=b+d, \quad \gamma=a-c,
$$

so that (6)-(7) become

$$
\theta^{\prime \prime}(\boldsymbol{t})+2 \alpha \theta^{\prime}(\boldsymbol{t})+\beta \theta(\boldsymbol{t})=\gamma, \quad \theta(0)=\theta_{0}, \quad \theta^{\prime}(0)=\theta_{0}^{\prime} .
$$

The technicalities related to solving this IVP have been relegated to the Appendix. The behaviour of the system modelled by (9) is controlled by the discriminant

$$
\Delta:=\alpha^{2}-\beta=\frac{(e+g)^{2}}{4}-(b+d)
$$

recall that the parameter $\alpha$ has the interpretation of sensitivity to the rate of change of certainty, while the parameter $\beta$ has the interpretation of sensitivity to the level of certainty.

Thus, if $\Delta=\alpha^{2}-\beta \neq 0$, the solution of the IVP (9) is

$$
\begin{aligned}
\theta(t)= & \frac{\gamma}{\beta}+\frac{\left(\theta_{0}-\frac{\gamma}{\beta}\right)\left(\alpha+\sqrt{\alpha^{2}-\beta}\right)+\theta_{0}^{\prime}}{2 \sqrt{\alpha^{2}-\beta}} \exp \left(\left(-\alpha+\sqrt{\alpha^{2}-\beta}\right) t\right) \\
& +\frac{\left(\theta_{0}-\frac{\gamma}{\beta}\right)\left(-\alpha+\sqrt{\alpha^{2}-\beta}\right)-\theta_{0}^{\prime}}{2 \sqrt{\alpha^{2}-\beta}} \exp \left(\left(-\alpha-\sqrt{\alpha^{2}-\beta}\right) t\right) .
\end{aligned}
$$

In the special case where $\Delta=\alpha^{2}-\beta<0$ and letting

$$
\omega=\sqrt{\beta-\alpha^{2}},
$$

it follows from (23) that the solution of the IVP (9) can be represented as

$$
\theta(t)=\frac{\gamma}{\beta}+e^{-\alpha t}\left[\left(\theta_{0}-\frac{\gamma}{\beta}\right) \cos \omega t+\frac{\left(\theta_{0}-\frac{\gamma}{\beta}\right) \alpha+\theta_{0}^{\prime}}{\omega} \sin \omega t\right] .
$$

In the special case where $\Delta=\alpha^{2}-\beta=0$, from (22) we get that the solution of the IVP (9) is 


$$
\theta(t)=\frac{\gamma}{\alpha^{2}}+e^{-\alpha t}\left[\left(\theta_{0}-\frac{\gamma}{\alpha^{2}}\right)+\left(\theta_{0}^{\prime}-\left(\frac{\gamma}{\alpha^{2}}-\theta_{0}\right) \alpha\right) t\right]
$$

\subsection{The general model}

We use the same notation as in the previous two subsections. We obtain the general model by aggregating the model of the objective certainty as in (3) with the model of the subjective certainty as in (11) about the coupling function, replacing the equilibrium constant $\bar{\theta}=(a-c) /(b+d)=\gamma / \beta$ in (11) with the objective certainty function as in (3). This is justified by the principle proposed by Hoch (1987), cited in Stanovich and West (1998) for the 'consensus effect' and explained in detail in Gheondea-Eladi (2016, Section 2.3). Thanks to the use of this type of aggregation and the delay between objective and subjective certainty, we are able to say that it is the subjective certainty that is responsible for the bifurcation between the oscillatory and non-oscillatory behaviour of the compound certainty. Hence, the behaviour of the system modelled by (9) is controlled by the discriminant $\Delta$ as in (10); recall that the parameter $\alpha$ has the interpretation of sensitivity to the rate of change of certainty, while the parameter $\beta$ has the interpretation of sensitivity to the level of certainty.

Thus, if the discriminant $\Delta=\alpha^{2}-\beta \neq 0$, from (3) and (11), we get the general mathematical model for the function that describes the EoC:

$$
\begin{aligned}
\theta(t)= & \frac{\gamma_{1}}{\beta_{1}}-\exp \left(-t \beta_{1}\right)\left(\frac{\gamma_{1}}{\beta_{1}}-\theta_{0,1}\right)+\frac{\left(\theta_{0}-\frac{\gamma}{\beta}\right)\left(\alpha+\sqrt{\alpha^{2}-\beta}\right)+\theta_{0}^{\prime}}{2 \sqrt{\alpha^{2}-\beta}} \exp \left(\left(-\alpha+\sqrt{\alpha^{2}-\beta}\right) t\right) \\
& +\frac{\left(\theta_{0}-\frac{\gamma}{\beta}\right)\left(-\alpha+\sqrt{\alpha^{2}-\beta}\right)-\theta_{0}^{\prime}}{2 \sqrt{\alpha^{2}-\beta}} \exp \left(\left(-\alpha-\sqrt{\alpha^{2}-\beta}\right) t\right),
\end{aligned}
$$

where, we use the notation

$$
\beta_{1}=b_{1}+d_{1}, \quad \gamma_{1}=a_{1}-c_{1} .
$$

If the discriminant $\Delta=\alpha^{2}-\beta<0$ we use the facts obtained at the end of Section 3.2. More precisely, with notation as in (12), we use the form of the evolution of subjective certainty as in (13) and (3) and get

$$
\theta(t)=\frac{\gamma_{1}}{\beta_{1}}-\exp \left(-t \beta_{1}\right)\left(\frac{\gamma_{1}}{\beta_{1}}-\theta_{0,1}\right)+e^{-\alpha t}\left[\left(\theta_{0}-\frac{\gamma}{\beta}\right) \cos (\omega t)+\frac{\theta_{0}^{\prime}+\alpha\left(\theta_{0}-\frac{\gamma}{\beta}\right)}{\omega} \sin (\omega t)\right]
$$

where $\omega=\sqrt{\beta-\alpha^{2}}$.

$$
\begin{aligned}
& \text { If } \Delta=0 \text {, from (3) and (14) we get } \\
& \qquad \theta(t)=\frac{\gamma_{1}}{\beta_{1}}-\exp \left(-t \beta_{1}\right)\left(\frac{\gamma_{1}}{\beta_{1}}-\theta_{0,1}\right)+e^{-\alpha t}\left[\left(\theta_{0}-\frac{\gamma}{\alpha^{2}}\right)+\left(\theta_{0}^{\prime}-\left(\frac{\gamma}{\alpha^{2}}-\theta_{0}\right) \alpha\right) t\right] .
\end{aligned}
$$

Based on this model, we carried out a computer simulation, see Figure 2, that emphasizes the three possible patterns. 


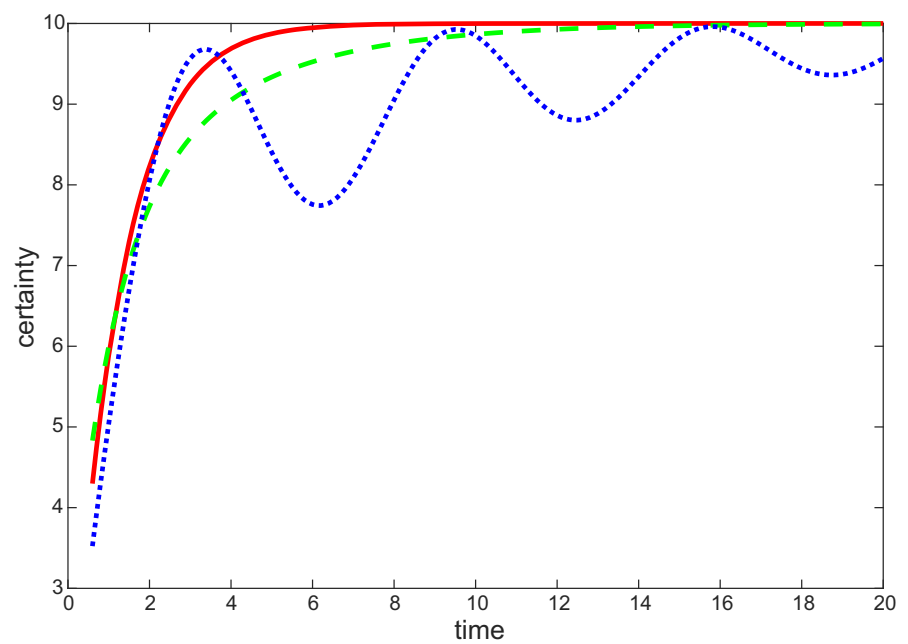

Figure 2. Simulation for the three general patterns of the evolution of certainty: $\Delta>0$ (solid line), $\Delta=0$ (dashed line), $\Delta<0$ (dotted line).

We performed computer simulations with varying parameter $\alpha$ (see (8)), in order to emphasize how the bifurcation appears when the discriminant is changing sign, for two different ways of analytically expressing formula (15). Due to computational limitations, in Figure 3 the simulation produces a singularity of the mesh at the critical value of $\alpha$ when $\Delta$ changes sign, while in Figure 4, the simulation produces no singularity of the mesh at any point, no matter whether $\alpha$ is critical or not. Formula (15) was used in the program for least-squares fitting. In the Appendix (see Proposition 5.2) we have proven the robustness

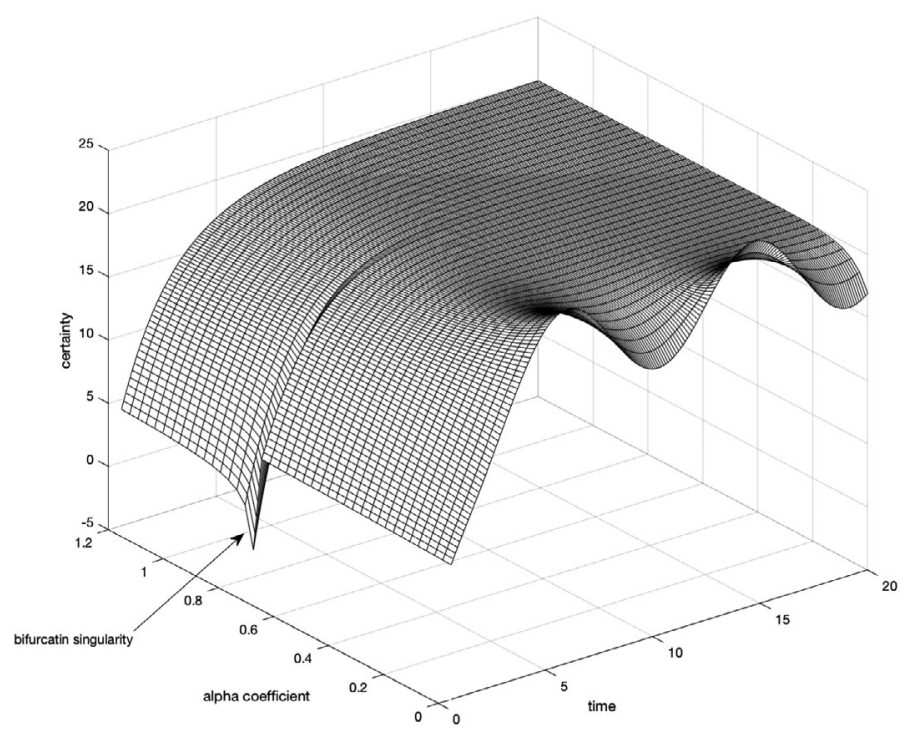

Figure 3. A simulation with varying parameter $\alpha$ showing the bifurcation point in the case when a singularity shows up. 


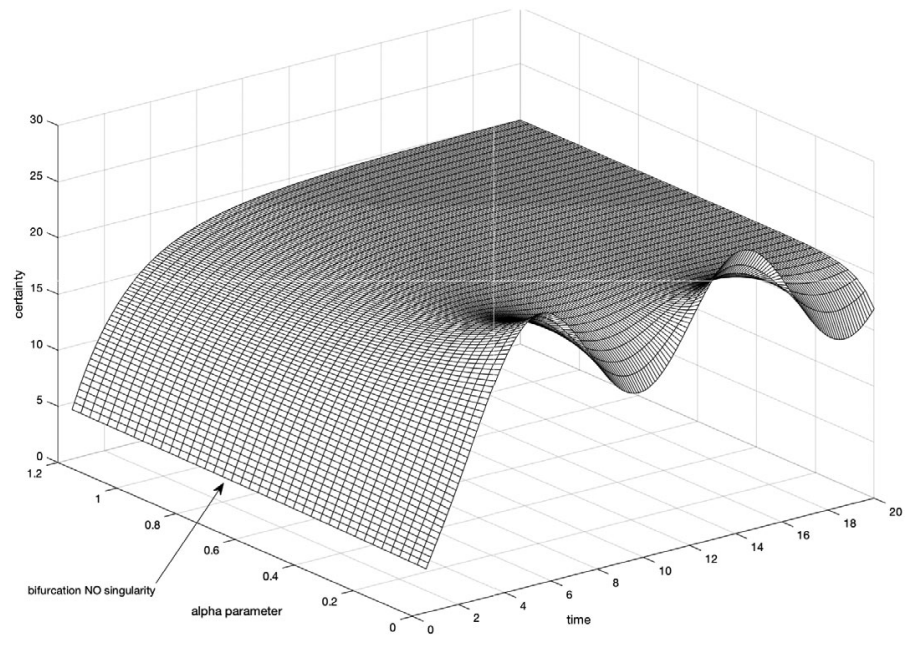

Figure 4. A simulation with varying parameter $\alpha$ showing the bifurcation point in the case when no singularity shows up.

of the latter formula, mathematically expressed as uniform continuity on compact sets with respect to all parameters.

In order to draw some conclusions from these representations, we have to distinguish the three separate cases corresponding to the value of the discriminant $\Delta=\alpha^{2}-\beta$ of the characteristic polynomial of the second-order IVP modelling the component corresponding to subjective certainty.

If the discriminant $\Delta$ is positive, we observe from (15) that we have evolution of the aggregated certainty to the equilibrium constant $\bar{\theta}$. In this case, no oscillation occurs. Similar conclusions can be drawn from (13) if $\Delta$ vanishes. Namely, when $\Delta=0$, we observe the same non-oscillatory form as when $\Delta>0$, except for a slight curve modification which is not relevant for the purposes of this paper.

If $\Delta<0$, it follows from (17) that the evolution of the aggregated certainty has the following characteristics. First, the aggregated certainty evolves to the equilibrium constant $\bar{\theta}=\gamma / \beta$. Second, the evolution to equilibrium occurs with a permanent oscillation with frequency $\omega \neq 0$.

In view of (8) and the receptor/provider functions as in (5), we obtain the interpretation of $\alpha^{2}$ as the sensitivity to the rate of change of certainty, expressed by the derivative $\theta^{\prime}$, while $\beta$ represents the sensitivity to the absolute level of certainty $\theta$. In order to substantiate this claim, we observe first that these are measurements at individual level and then that, at the inner individual level, $e$ and $g$ are the providers' and receptors' sensitivities to the rate of change of certainty, while $b$ and $d$ are the providers' and receptors' sensitivities to the absolute value of certainty. Taking into account that the bifurcation appears as $\Delta=\alpha^{2}-\beta$ changes sign, this means that the bifurcation shows which one of these two sensitivities prevails. If the sensitivity to the rate of change of certainty is higher, oscillation of the certainty level does not appear, while if the sensitivity to the absolute level of certainty is higher, oscillation of the certainty level appears. 


\section{Data and analysis}

In order to test the model proposed in Section 3 we employ, with permission, the data collected in the study by Gheondea-Eladi (2016). We briefly summarize the data collection method and then present the numerical procedure employed to analyse it, as well as the empirical results of the data analysis.

\section{I. Data collection and measurement}

Ninety-seven students of sociology and social work, in groups of 4-6, were presented with the NASA Moon survival task (Teleometrics International, 2007):

You are a member of a crew scheduled to meet the mother-ship on the bright face of the Moon. Due to some malfunction your ship was forced to land 322 kilometers away from the meeting point. During the landing procedure, most of the equipment on board was destroyed. Since your survival depends upon reaching the mother-ship, you have to choose the most important items available in order to walk the distance to the mother-ship. There are 15 items left intact after the impact. Your mission is to order them according to their importance such that your crew will reach the meeting point. Number with 1 the most important item and with 10 the least important item.

- Match box

- Condensed food

- 15 meters of nylon thread

- Parachute silk

- Portable heating unit

- Two 45 caliber pistols

- 1 box of condensed milk

- 2 oxygen tanks of $45 \mathrm{~kg}$ each

- Stellar map (around the Moon)

- Automatic inflation rescue vest

- Magnetic compass

- 19 liters of water

- Signaling missiles

- First aid kit including needles and a syringe

- Radio emitter and transmitter with solar batteries

Each group member solved the task individually, indicating a solution, in the form of an individual hierarchy and its certainty level, and then discussed it with the group. Participants were asked to write each perceived change in their individual solution as follows: the new solutions, the time at which each new solution occurred, the certainty level of the new solution itself and the certainty of the new solution compared to the initial individual one. Participants were asked to report these changes as soon as they perceived them. After the group discussion had reached a consensus or when consensus was considered to be impossible, each participant was asked to individually write the group solution, write how certain they were about the group decision, write how satisfied they were with the way the decision was made, and how interesting they found the decision problem.

Participants' experimental output comprised of two time series, one corresponding to the certainty of the new decision with respect to the initial individual decision (the 
reference point salient certainty measurement, here called CII) and the other corresponding to the certainty of the new solution itself (the common certainty measurement, here called CIF). Since participants in the pre-test reported the certainty evaluations with different reference points, we employed a procedure proposed by Hoch (1987), cited in (Stanovich \& West, 1998), for the 'certainty effect' and adapted it to the current case by using the CII and CIF to derive, by subtraction, the absolute individual certainty (called CI). This procedure is explained in detail in Section 2.3 of (Gheondea-Eladi, 2016). At each moment in time during the group discussion CII was measured through the following question: 'How certain are you (now) of the initial hierarchy?' CIF was measured through the question: 'How certain are you (now) of the new hierarchy?' Both CII and CIF are measured repeatedly during the group discussion. These differences were verbally explained and exemplified in the experimental instructions. Participants received verbal clarification, where necessary, that they should indicate for CII, 'at this time and after the discussion you had so far, how certain are you of the initial hierarchy', while for CIF they should indicate, 'at this time and after the discussion you had so far, how certain are you of the new hierarchy you have just indicated'. In the pre-test, two different experimental tasks and phrasings for these questions were tested before this final form was reached based upon post-experimental discussions with the participants and analysis of the pretest results.

For the model to be tested it was important that the communication in the group discussion could be approximated by time, and consequently required a single information unit to be communicated at a time. After removing participants who indicated more than one change at a time, the sample size was 39 (for details, see section 3.3 in Gheondea-Eladi, 2016). However, 21 of the participants who indicated more than one change at a time reported this only once or twice. In such cases, without much loss of information, these instances could be removed from the time series, leaving an individual time series sample of acceptable length to be studied. Therefore, unlike in the previous research, the sample employed here numbers 60 participants. Also in contrast to the previous paper, we discarded the instances in the time series where one of the measurements (CII or CIF) had missing data, thus leaving the size of the time series smaller than previously (from a maximum of $N=20$ to 16). This operation was performed because in this paper we analyse all measurements (CII, CIF and CI) in a single instance, as opposed to separately as performed previously, and the algorithm required the same length of the time series for all three measurements.

\subsection{Numerical procedure}

Each participant yielded a time series, represented by two sequences of data:

$$
x \text { data }=\left(x_{1}, x_{2}, x_{3}, \ldots, x_{N}\right), \quad y \text { data }=\left(y_{1}, y_{2}, y_{3}, \ldots, y_{N}\right),
$$

where $N$ is the time series size, $x_{j}$ is the time sequence expressed in seconds, and $y_{j}$ is the reported level of certainty corresponding to time $x_{j}$. $N$ has values between 11 and 16 and $y_{j}$ has values between -7 and 7 , since it represents the data collected (CII and CIF) and derived (CI).

The least-squares approximation method was employed, under the conditions required for time series (i.e. error is spherical according to Beck and Katz (2007)) by using lsqcurvefit in MATLAB. As in the previous analysis (Gheondea-Eladi, 2016),, the 
Euclidean distance between the general model in (17) and the data series in $\mathbb{R}^{N}$ is minimized.

The algorithm searches for a vector $c=\left(c_{j}\right)_{j=1}^{8}$, within a confidence interval $\left[c_{j}^{\min }, c_{j}^{\max }\right]$, for each $j=1, \ldots, 8$, where, with respect to the notation as in the general formula (15), the parameters are identified as follows:

$$
c_{1}=\alpha, c_{2}=\beta, c_{3}=\gamma, c_{4}=\theta_{0}, c_{5}=\theta_{0}^{\prime}, c_{6}=\beta_{1}, c_{7}=\gamma_{1}, c_{8}=\theta_{0,1} .
$$

\subsection{Empirical results}

Based on the numerical procedure described previously, the model proposed in Section 3 has been tested against the data collected. Moreover, in this section we show that:

1. the bifurcation, the split in participants' behaviour, appears irrespective of the starting point provided;

2. participants' EoC appears with two different evolution patterns.

In order to verify the first conjecture, we aim to show that even if the algorithm begins to search from a point on the bifurcation line $(\Delta=0)$ or either side of it (either $\Delta<0$ or $\Delta>0$ ), this leads to similar results for each of the collected data (CII, CIF and CI). Thus, we have generated the $c$ coefficients based on the two relevant cases, $\Delta<0, \Delta>0$, when $\alpha>0$, and $\beta>0$ (see (8)). In Table 2 , the frequency of approximations resulting in $\Delta<0$ given that the shooting data had $\Delta=0$ is 47 . Only three more of the lsqcurvefit approximations for CII and two for CIF ended up having $\Delta<0$ when the initial conditions were on the separation plane, $\Delta=0$, than when they were in the area described by $\Delta<0$. For the absolute certainty level, no difference was observed between approximations with the two different initial conditions.

Having revealed analytically (Section 3) and empirically (above) that the bifurcation exists, we will show that participants' EoC exhibits two different evolution patterns by providing the frequency of participants in each group: oscillation for those for whom the sensitivity to the absolute value of certainty prevails $(\Delta<0)$ and evolution to equilibrium for those for whom the sensitivity to its rate of change prevails $(\Delta>0)$. In Table 2 , for the two direct certainty measurements (CII and CIF), these groups are clearly differentiated. If measured by making salient the reference point (CII), between 44 and 47 participants have an EoC which oscillates to equilibrium and the rest have an $\mathrm{EoC}$ which does not oscillate. If measured without making salient the initial reference point (CIF), between 33

Table 2. Frequency of coefficients with initial conditions $\Delta<0$ and $\Delta>0$ reveal the bifurcation irrespective of where the shooting data is situated. Shooting data is the initial point for the algorithm iterations, situated within a certain confidence interval for each parameter; the shooting data is derived empirically

\begin{tabular}{|c|c|c|c|c|c|c|}
\hline \multirow[b]{2}{*}{ Shooting Data } & \multicolumn{2}{|c|}{ CII } & \multicolumn{2}{|c|}{ CIF } & \multicolumn{2}{|c|}{$\mathrm{CI}$} \\
\hline & $\Delta<0$ & $\Delta>0$ & $\Delta<0$ & $\Delta>0$ & $\Delta<0$ & $\Delta>0$ \\
\hline$\Delta<0$ & 44 & 16 & 25 & 35 & 58 & 2 \\
\hline$\Delta>0$ & 47 & 13 & 27 & 33 & 58 & 2 \\
\hline
\end{tabular}




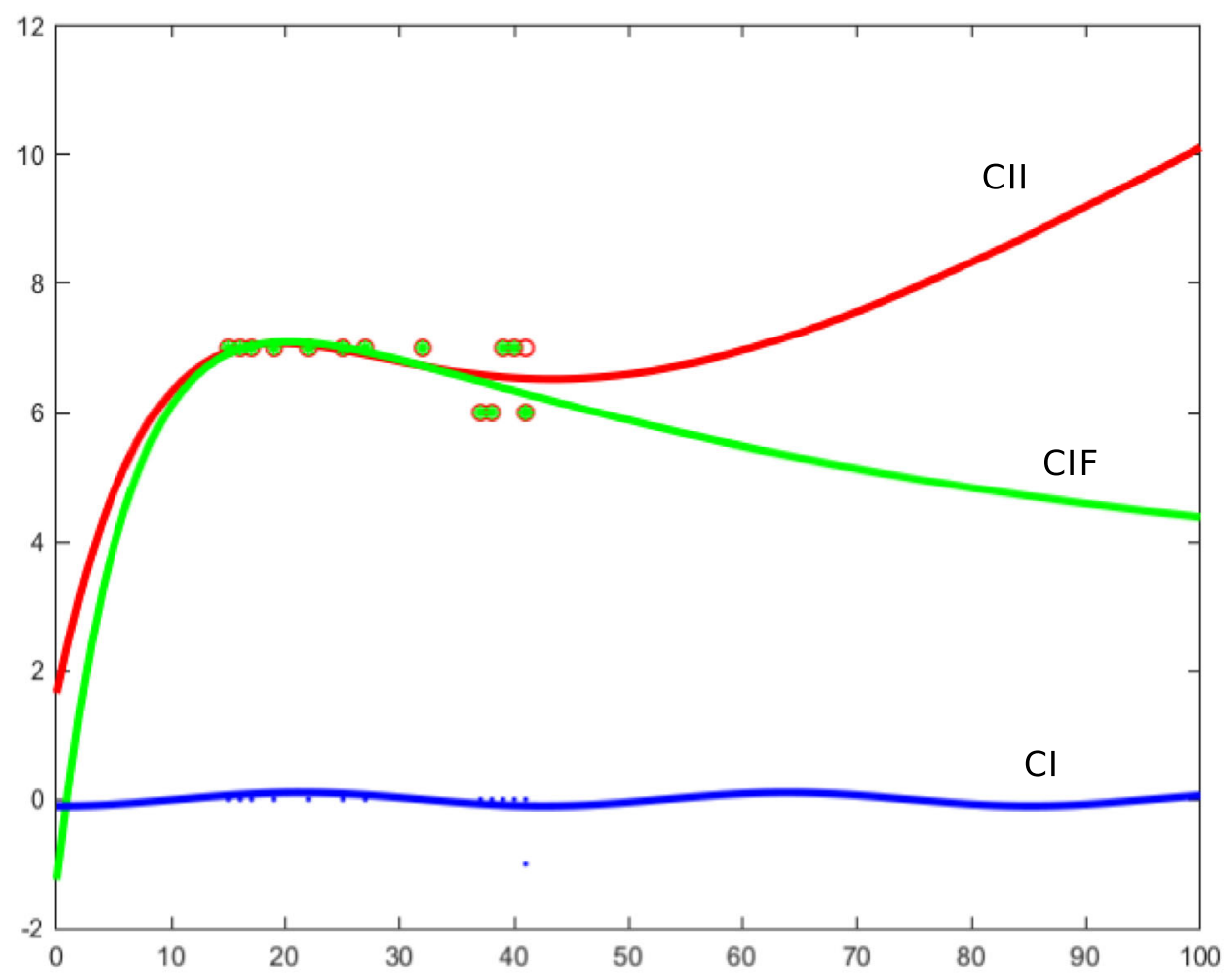

Figure 5. Participant 1: data for CI (points), CII (circle), and CIF (star) and the theoretical model curve fit for each.

and 35 participants reveal evolution to equilibrium without oscillation and the rest have an EoC which oscillates to equilibrium. Because $\mathrm{CI}$ is a comparative measure, computed as the difference between CII and CIF, the particularities of individual EoC, such as those revealed by the observed split, will not be visible for this measurement. Therefore, if considering the CI measurement, participants' EoC is homogeneous.

For exemplification, we include the graphical representation of the family of functions described by our model in Figure 2, and for two participants, based on their data, in Figures 5 and 6.

\section{Discussion and conclusions}

In this paper the evolution of certainty in small-group decisions by consensus reveals two different patterns, delimited by a bifurcation in the theoretical model. We suggest that this bifurcation differentiates two groups: those sensitive to the absolute value of certainty and those sensitive to its rate of change. We have also reviewed alternative interpretations of this behaviour in the form of certainty/uncertainty orientation, certainty interpretation, and intuition and affect related to certainty/uncertainty. Experimental evidence has shown that the observed bifurcation in the new model proposed is not due to the numerical procedure and curve-fitting algorithm used. 


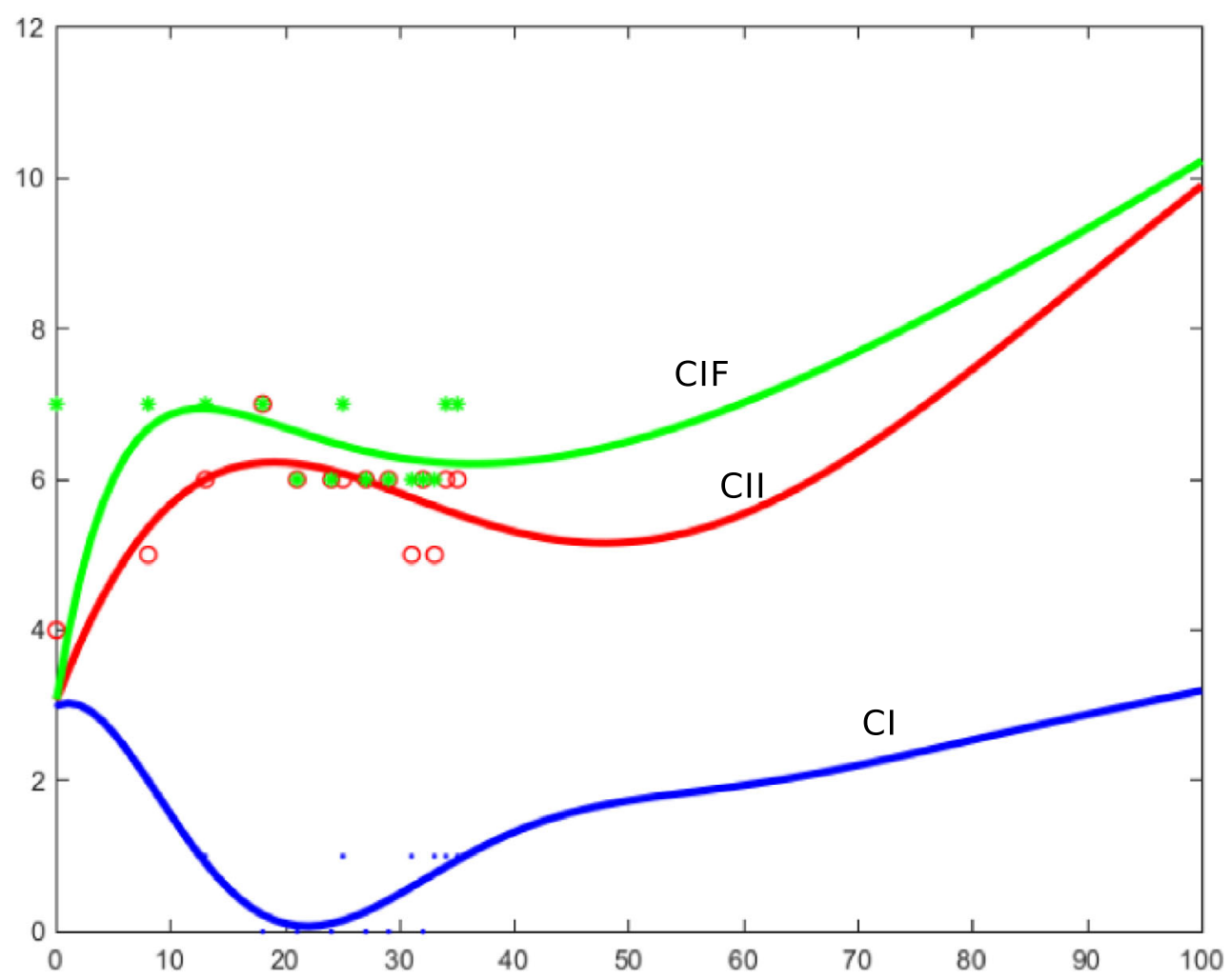

Figure 6. Participant 2: data for CI (points), CII (circle), and CIF (star) and the theoretical model curve fit for each.

\section{I. Limitations and questions}

Some observations are in order regarding the limitations of this analysis. First, we cannot yet provide a sociopsychological reason for $\alpha$ as the average of the receptors' and providers' sensitivities to the rate of change of certainty and $\beta$ as the sum of the sensitivities to its absolute value. We can only explain why the relationship between the sensitivity to the absolute value of certainty and its rate of change is of the form $f(x)=x^{2}$ : this is because the units of measurement of $\alpha$ and of $\beta$ are different. Second, based on this analysis it is not possible to say anything about the relationship between the frequencies of the group for which the EoC is oscillating to equilibrium and that for which it is not oscillating. If the distribution could be assumed uniform, we might expect to find that, in general, there are more people for whom the EoC has $\Delta<0$ than $\Delta>0$. Further research might investigate the characteristics of this distribution. Without claiming generalizability, our results suggest that emphasizing the reference point for certainty may lead to different frequency ratios between the two groups, than when the certainty measurement does not make the reference point salient. Future studies should continue to control for this aspect. Third, in this paper we proposed an interpretation of the variables leading to bifurcation based on their role within this model and reviewed other possible interpretations: certainty/ uncertainty orientation, uncertainty interpretation as danger or opportunity, and certainty/uncertainty affect and intuition. Future research should further investigate the link between the interpretation of the variables relevant to this split in the EoC and the 
interpretations proposed in the literature review, by controlling for the level of abstraction.

\subsection{Implications and future studies}

There are two main implications of this study for management. First, future studies might investigate the optimum group decision time necessary to reach the equilibrium certainty. This would be particularly important in order to avoid closing the group discussion before the equilibrium value is reached. Second, it is important to notice that closing group discussion before the optimum time might lead to cases of over- and underconfidence with respect to the equilibrium value.

Furthermore, there are implications of these findings for psychological research. Given that the current model interprets the balance between the absolute value of certainty and the change in certainty, it may be possible to devise methodologies that manipulate these variables experimentally and observe the type of EoC.

Based on the results obtained so far, it will be possible to further explore models which take into account the influences exerted by individuals within the group discussion. In this and the previous research we have assumed that none of the group members make any attempt to artificially elevate the level of certainty of the other members. When a forcing factor is considered, the model for the evolution of certainty has a higher degree of complexity. At this point, studying forced decisions of the evolution of certainty in group decisions will help us identify influenced decisions when this influence is not necessarily a product of more knowledge, but an artificial elevation of members certainty or selfconfidence, irrespective of the level of information available. In this way a future model can touch upon the issues of consensus building through manipulation of the certainty level.

\section{Acknowledgements}

The data used for this paper is available in the online Supplementary Materials, together with the code used to analyse the data.

\section{Conflicts of interest}

All authors declare no conflict of interest.

\section{Author contributions}

Alexandra Gheondea-Eladi (Conceptualization; Data curation; Investigation; Software; Writing - original draft; Writing - review \& editing) Aurelian Gheondea (Conceptualization; Formal analysis; Software; Writing - original draft; Writing - review \& editing).

\section{References}

Beck, N., \& Katz, J. N. (2007). Random coefficient models for time-series-cross-section data: Monte Carlo experiments. Political Analysis, 15, 182-195.

Beek, P. J., Verschoor, F., \& Kelso, S. (1997). Requirements for the emergence of a dynamical social psychology. Psychological Inquiry, 8, 100-104. 
Betsch, C. (2008). Chronic preferences for intuition and deliberation in decision making: Lessons learned about intuition from an individual differences approach. In H. Plessner, C. Betsch \& T. Betsch (Eds.), Intuition in judgment and decision-making, chapter 11 (pp. 231-248). New York, NY: Lawrence Erlbaum Associates.

Betsch, T., \& Glöckner, A. (2010). Intuition in judgment and decision making: Extensive thinking without effort. Psychological Inquiry, 21, 279-294.

Birch, D. (2009). The dynamics of the stream of behavior. Journal of Mathematical Psychology, 53 , 106-118.

Bixtera, M. T., \& Luhmann, C. C. (2020). Delay discounting in dyads and small groups: Group leadership, status information, and actor-partner interdependence. Journal of Experimental Social Psychology, 86, 1-10.

Bonito, J. A., Ervin, J. N., \& Staggs, S. M. (2016). Estimation and application of the latent group model. Group Dynamics: Theory, Research and Practice, 20, 126-143.

Carver, C. S. (1997). Dynamical social psychology: Chaos and catastrophe for all. Psychological Inquiry, 8, 110-121.

Chemers, M. M. (2004). Leadership effectiveness: Functional, contructivist and empirical perspectives. In D. Van Knippenberg \& M. Hogg (Eds.), Leadership and power: Identity processes in groups and organizations, chapter 2. (5-17). Gateshead: SAGE Publications Ltd.

De Dreu, C. K., Nijstad, B. A., \& van Knippenberg, D. (2008). Motivated information processing in group judgment and decision making. Personality \& Social Psychology Research, 12(1), 22-49.

de Kwaadsteniet, E. W., \& van Dijk, E. (2010). Social status as a cue for tacit coordination.Journal of Experimental Social Psychology, 46, 515-524.

Deffuant, G., Neau, D., Amblard, F., \& Weisbuch, G. (2000). Mixing beliefs among interacting agents. Advanced Complex Systems, 3, 87-98.

Diamond, L. M. (1986). The desire disorder in research on sexual orientation in women: Contributions of dynamical systems theory. Archives of Sexual Behavior, 41, 73-83.

Diamond, L. M. (2007). A dynamical systems approach to the development and expression of female same-sex sexuality. Perspectives on Psychological Science, 2, 142-163.

Duncan, S. M., Wengrovitz, S. M., Sedlovskaya, A., \& Patalano, A. L. (2007). Weighing waiting: The influence of information certainty and delay penalty on waiting for noninstrumental information. Judgment and Decision Making, 2, 351-358.

Dunning, D., Griffin, D. W., Milojkovic, J. D., \& Ross, L. (1990). The overconfidence effect in social prediction. Journal of Personality and Social Psychology, 58, 568-581.

Eiser, R. J., \& Bhavnani, K.-K. (1994). The effect of situational meaning on the behaviour of subjects in the Prisoner's Dilemma Game. European Journal of Social Psychology, 4(1), 93-97.

Epstein, S. (2008). Intuition from the perspective of cognitive-experiential self-theory. In P. Henning, C. Betsch \& T. Betsch (Eds.), Intuition in judgment and decision-making, chapter 2 (pp. 23-38). New York, NY: Lawrence Erlbaum Associates, Publishing.

Estrada, E., \& Vargas-Estrada, E. (2013). How peer pressure shapes consensus, leadership and innovations in social groups. Nature Scientific Reports, 3, 1-6.

Evans, A., \& Clark, J. (2012). Source characteristics and persuasion: The role of self-monitoring in self-validation. Journal of Experimental Social Psychology, 48, 383-386.

Fidler, K., \& Kareev, Y. (2006). Does decision quality (always) increase with the size of information samples? Some vicissitudes in applying the law of large numbers. Journal of Experimental Psychology: Learning, Memory, and Cognition, 32, 883-903.

Fraser, C. (1971). Group risk-taking and group polarization. European Journal of Social Psychology, I, 493-510.

Friedkin, N., \& Johnsen, E. (1990). Social influence and opinions. Journal of Mathematical Sociology, 15, 193-206.

Gheondea-Eladi, A. (2016). The evolution of certainty in a small decision-making group by consensus. Group Decision and Negotiation, 25, 127-155. 
Giardini, F., Coricelli, G., Joffily, M., \& Sirigu, A. (2008). Overconfidence in predictions as an effect of desirability bias. In M. Abdellaoui, \& J. Hey (Eds.), Advances in decision making under risk and uncertainty. (163-180). Berlin, Germany: Springer.

Gigerenzer, G., \& Brighton, H. (2009). Homo Heuristicus: Why biased minds make better inferences. Topics in Cognitive Science, 1, 107-143.

Gigerenzer, G., \& Gaissmaier, W. (2011). Heuristic decision making. Annual Review of Psychology, $62,451-482$.

Hertwig, R. (2012). The psychology and rationality of decisions from experience. Synthese, 187, 269-292.

Hertwig, R., \& Pleskac, T. (2010). Decisions from experience: Why small samples? Cognition, 115, $225-237$.

Hinsz, V. B., Tindale, R. S., \& Vollrath, D. A. (1997). The emerging conceptualization of groups as information processors. Psychological Bulletin, 121(1), 43-64.

Hirokawa, R. Y., \& Poole, S. M. (1996). Communication and group decision making (2nd ed.). Thousand Oaks, CA: Sage Publications.

Hoch, S. J. (1987). Perceived consensus and predictive accuracy: The pros and cons of projection. Journal of Personality and Social Psychology, 53, 221-234.

Hodson, G., \& Sorrentino, R. M. (1997). Groupthink and uncertainty orientation: Personality differences in reactivity to the group situation. Group Dynamics: Theory, Research and Practice, 1, 144-155.

Hoffman, H., Granhag, P., Kwong See, S., \& Loftus, E. (2001). Social influences on reality-monitoring decisions. Memory and Cognition, 29, 394-404.

Hosenfeld, B., Bos, E., Wardenaar, K., Conradi, H., van der Maas, H., Visser, I., \& de Jonge, P. (2015). Major depressive disorder as a nonlinear dynamic system: Bimodality in the frequency distribution of depressive symptoms over time. BMC Psychiatry, 15, 1-9.

Huber, G. L., Sorrentino, R. M., Davidson, M. A., Eppler, R., \& Roth, J. W. (1992). Uncertainty orientation and cooperative learning: Individual differences within and across cultures. Learning and Individual Differences, 4(1), 1-24.

Jiao, Y., \& Li, Y. (2021). An active opinion dynamics model: The gap between the voting result and group opinion. Information Fusion, 65, 128-148.

Johnson, M. P., \& Ewens, W. (1971a). Power relations and affective style as determinants of confidence in impression formation in a game situation. Journal of Experimental Social Psychology, 7, 98-110. https://doi.org/10.1016/0022-1031(71)90059-X

Johnson, M. P., \& Ewens, W. (1971b). Power relations and affective style as determinants of impression formation. Journal of Experimental Social Psychology, 7, 98-110.

Kahneman, D., Slovic, P., \& Tversky, A. (1982). Judgment under uncertainty: Heuristics and biases. New York, NY: Cambridge University Press.

Kang, Y. (2009). Role of health locus of control between uncertainty and uncertainty appraisal among patients with atrial fibrillation. Western Journal of Nursing Research, 31, 187-200.

Koopmans, M., \& Stamovlasis, D. (Eds.) (2016). Complex dynamical systems in education: Concepts, methods and applications. New York, NY: Springer.

Larson, R. J., Tindale, R., \& Yoon, Y. (2019). Advice taking by groups: The effects of consensus seeking and member opinion differences. Group Processes \& Intergroup Relations, 23, 921 942. https://doi.org/10.1177/1368430219871349

Locke, C., \& Anderson, C. (2015). The downside of looking like a leader: Power, nonverbal confidence and participative decision-making. Journal of Experimental Social Psychology, 58 , $42-47$.

Lynch, S. (2004). Dynamical systems with applications using MATLAB. New York, NY: Springer.

Medio, A., \& Lines, M. (2001). Nonlinear dynamics: A primer. Cambridge, UK: Cambridge University Press.

Mishel, M. H. (1990). Reconceptualization of the uncertainty in illness theory. State of the Science, $22,256-262$. 
Oskamp, S. (1982). Overconfidence in case-study judgments. In D. Kahneman, P. Slovic \& A. Tversky (Eds.), Judgment under uncertainty: Heuristics and biases. New York, NY: Cambridge University Press.

Pham, V.-T., Vaidyanathan, S., Volos, C., \& Kapitaniak, T. (2018). Nonlinear dynamical systems with self-excited and bidden attractors. Berlin, Germany: Springer.

Plessner, H., Betsch, C., \& Betsch, T. (Eds.) (2008). Intuition in judgment and decision making. New York, NY: Lawrence Erlbaum Associates, Publishing.

Plous, S. (1995). A comparison of strategies for reducing interval overconfidence in group judgments. Journal of Applied Psychology, 80, 443-454.

Poole, M. S. (1983). Decision development in small groups II: A study of multiple sequences in decision making. Communication Monographs, 50, 206-232.

Punchochar, J., \& Fox, P. (2004). Confidence in individual and group decision-making: When "Two Heads" are worse than one. Journal of Educational Psychology, 96, 582-591.

Rinne, L. F., \& Mazzocco, M. M. M. (2013). Inferring uncertainty from interval estimates: Effects of alpha level and numeracy. Judgment and Decision Making, 8, 330-344.

Sadovnichiy, V. A., \& Zgurovsky, M. Z. (1986). Advances in dynamical systems and control. Berlin, Germany: Springer.

Shuper, P. A., Sorrentino, R. M., Otsubo, Y., Hodson, G., \& Walker, M. A. (2004). A theory of uncertainty orientation implications for the study of individual differences within and across cultures. Journal of Cross-Cultural Psychology, 35, 460-480.

Shuping, W., Wang, F., \& Dong, J. (2018). A group decision making method with interval valued fuzzy preference relations based on the geometric consistency. Information Fusion, 40, 87100.

Slevin, D. P., Boone, L. W., Russo, E. M., \& Allen, R. S. (1998). CONFIDE: A collective decision-making procedure using confidence estimates of individual judgements. Group Decision and Negotiation, 7, 179-194.

Sorrentino, R. M., Bobocel, D. R., Gitta, M. Z., Olson, J. M., \& Hewitt, E. C. (1988). Uncertainty orientation and persuasion: Individual differences in the effects of personal relevance on social judgments. Journal of Personality and Social Psychology, 55, 357-371.

Sorrentino, R. M., \& Brouwers, M. C. (1993). Uncertainty orientation and protection motivation theory: The role of individual differences in health compliance. Personality Processes and Individual Differences, 65(1), 102-112.

Sorrentino, R. M., Holmes, J. G., Hanna, S. E., \& Sharp, A. (1995). Uncertainty orientation and trust in close relationships: Individual differences in cognitive styles. Personality Processes and Individual Differences, 68, 314-327.

Sorrentino, R. M., Smithson, M., Hodson, G., Roney, C. J., \& Walker, A. M. (2003). The theory of uncertainty orientation: A mathematical reformulation. Journal of Mathematical Psychology, $47,132-149$.

Staggs, S. M., Bonito, J. A., \& Ervin, J. N. (2018). Measuring and evaluating convergence processes across a series of group discussions. Group Decision and Negotiation, 27, 715-733.

Stankov, L., Morony, S., \& Lee, Y. P. (2014). Confidence: The best non-cognitive predictor of academic achievement? Educational Psychology, 34(1), 9-28.

Stanovich, K. E., \& West, R. F. (1998). Individual differences in rational thought. Journal of Experimental Psychology: General, 127, 161-188.

Teleometrics International. (2007). NASA Moon survival task.

Thaler, R. H. (1985). Mental accounting and consumer choice. Marketing Science, 4, 199-214.

Thaler, R. H. (1999). Mental accounting matters. Journal of Behavioral Decision Making, 12, $183-$ 206.

Thorson, K., Dumitru, O., Mendes, B., \& West, T. (2019). Influencing the physiology and decisions of groups: Physiological linkage during group decision-making. Group Processes and Intergroup Relations, 24(1), 1-15.

Unal, R., Keating, C., Chytka, T., \& Conway, B. (2005). Calibration of expert judgments applied to uncertainty assessment. Engineering Management Journal, 12, 34-43. 
Vallacher, R. R., \& Nowak, A. (1997). Dynamical social psychology: The next interation. Psychological Inquiry, 8, 152-160.

Vallacher, R. R., Read, S. J., \& Nowak, A. (2002). The dynamical perspective in personality and social psychology. Personality and Social Psychology Review, 6, 264-273.

van den Bos, K., \& Lind, A. E. (2002). Uncertainty management by means of fairness judgments. Advances in experimental social psychology, 34, 1-60.

Votruba, H., \& Kwan, V. S. (2015). Disagreeing on whether agreement is persuasive: Perceptions of expert group decisions. PLoS One, 10, e0121426.

Weber, E. U., \& Lindemann, P. G. (2008). From intuition to analysis: Making decisions with your head, your heart, or by the book. In P. Henning, C. Betsch \& T. Betsch (Eds.), Intuition in judgment and decision-making, chapter 12 (pp. 191-208). New York, NY: Lawrence Erlbaum Associates, Publishing.

Wichman, A., Brinol, P., Petty, R., Rucker, D., Tormala, Z., \& Waery, G. (2009). Doubting one's doubt: A formula for confidence? Journal of Experimental Social Psychology, 46, 350-355. https://doi.org/10.1016/j.jesp.2009.10.012

Yu, W., Zhang, Z., \& Zhong, Q. (2018). Consensus reaching for MAGDM with multi-granular hesitant fuzzy linguistic term sets: A minimum adjustment-based approach. Annals of Operations Research, 300, 443-466.

Zamfir, C. (2005). Incertitudinea - o perspectiväf psibosociologicÄf [Uncertainty - a psychsociological perspective]. Bucureåÿti: Editura Economicäf.

Zarnoth, P., \& Sniezek, J. A. (1997). The social influence of confidence in group decision making. Journal of Experimental Social Psychology, 33, 345-366.

Zhang, H., Zhao, S., Kou, G., Li, C.-C., Dong, Y., \& Herrera, F. (2020). An overview on feedback mechanisms with minimum adjustment or cost in consensus reaching in group decision making: Research paradigms and challenges. Information Fusion, 60, 65-79. https://doi.org/10.1016/j. inffus.2020.03.001

Zhang, Z., Gao, Y., \& Li, Z. (2020). Consensus reaching for social network group decision making by considering leadership and bounded confidence. Knowledge Based Systems, 204, 106240. https://doi.org/10.1016/j.knosys.2020.106240

Zhao, Y., Zhang, L., Tang, M., \& Kou, G. (2016). Bounded confidence opinion dynamics with opinion leaders and environmental noises. Computers and Operations Research, 74, 205-213.

Zhu, D. (2014). Group polarization in board decisions about CEO compensation. Organization Science, 25, 552-571.

Received 30 June 2020; revised version received 8 April 2021

\section{Supporting Information}

The following supporting information may be found in the online edition of the article:

Appendix S1. Data

Appendix S2. MATLAB Codes 


\section{Appendix :}

\section{Some technical results}

We consider the second-order initial value problem (IVP)

$$
y^{\prime \prime}(t)+2 \alpha y^{\prime}(t)+\beta y(t)=\gamma, y(0)=u, y^{\prime}(0)=v
$$

where $\alpha, \beta>0$ and $\gamma, u, v, t \in \mathbb{R}$.

Proposition 5.1. The IVP (A1) has unique solution defined as follows: if $\alpha^{2}-\beta \neq 0$ then

$$
\begin{aligned}
y(t)= & \frac{\gamma}{\beta}+\frac{\left(u-\frac{\gamma}{\beta}\right)\left(\alpha+\sqrt{\alpha^{2}-\beta}\right)+v}{2 \sqrt{\alpha^{2}-\beta}} \exp \left(\left(-\alpha+\sqrt{\alpha^{2}-\beta}\right) t\right) \\
& +\frac{\left(u-\frac{\gamma}{\beta}\right)\left(-\alpha+\sqrt{\alpha^{2}-\beta}\right)-v}{2 \sqrt{\alpha^{2}-\beta}} \exp \left(\left(-\alpha-\sqrt{\alpha^{2}-\beta}\right) t\right) ;
\end{aligned}
$$

and if $\alpha^{2}-\beta=0$ then

$$
y(t)=\frac{\gamma}{\alpha^{2}}+e^{-\alpha t}\left[\left(u-\frac{\gamma}{\alpha^{2}}\right)+\left(v-\left(\frac{\gamma}{\alpha^{2}}-u\right) \alpha\right) t\right] .
$$

In addition, if $\alpha^{2}-\beta<0$ then, letting $\omega=\sqrt{\beta-\alpha^{2}}$, the solution $y$ defined as in (A2) has the following representation:

$$
y(t)=\frac{\gamma}{\beta}+e^{-\alpha t}\left[\left(u-\frac{\gamma}{\beta}\right) \cos \omega t+\frac{\left(u-\frac{\gamma}{\beta}\right) \alpha+v}{\omega} \sin \omega t\right] .
$$

Proof. Since (A1) is a second-order linear ODE with constant coefficients and nonhomogeneous, it has a unique global solution which is obtained as the sum of the constant solution $y_{p}(1)=\gamma / \beta$ and the general solution $y_{g}$ of the associated homogeneous ODE,

$$
y^{\prime \prime}+2 \alpha y^{\prime}+\beta=0
$$

Let $\lambda_{1}$ and $\lambda_{2}$ be the roots of the characteristic polynomial

$$
p(\lambda)=\lambda^{2}+2 \alpha \lambda+\beta
$$

that is,

$$
\lambda_{1,2}=-\alpha \pm \sqrt{\alpha^{2}-\beta}
$$

and let the discriminant be denoted by 


$$
\Delta=\alpha^{2}-\beta
$$

We have to treat separately the cases of positive, negative, and null discriminant. is

If $\Delta>0$ then the roots $\lambda_{1}$ and $\lambda_{2}$ are real and distinct and the general solution $y_{g}$ of (A5)

$$
y_{\mathrm{g}}(t)=C_{1} \exp \left(\left(-\alpha+\sqrt{\alpha^{2}-\beta}\right) t\right)+C_{2} \exp \left(\left(-\alpha-\sqrt{\alpha^{2}-\beta}\right) t\right)
$$

Hence, the general solution of (20) is

$$
y(t)=\frac{\gamma}{\beta}+C_{1} \exp \left(\left(-\alpha+\sqrt{\alpha^{2}-\beta}\right) t\right)+C_{2} \exp \left(\left(-\alpha-\sqrt{\alpha^{2}-\beta}\right) t\right) .
$$

From the initial conditions $\mathrm{y}(0)=\mathrm{u}$ and $\mathrm{y}^{\prime}(0)=\mathrm{v}$ we obtain the system with unknowns $\mathrm{C}_{1}$ and $\mathrm{C}_{2}$

$$
\begin{gathered}
C_{1}+C_{2}=u-\frac{\gamma}{\beta}, \\
\left(-\alpha+\sqrt{\alpha^{2}-\beta}\right) C_{1}+\left(-\alpha-\sqrt{\alpha^{2}-\beta}\right) C_{2}=v,
\end{gathered}
$$

with solution

$$
\begin{gathered}
C_{1}=\frac{\left(u-\frac{\gamma}{\beta}\right)\left(\alpha+\sqrt{\alpha^{2}-\beta}\right)+v}{2 \sqrt{\alpha^{2}-\beta}}, \\
C_{2}=\frac{\left(u-\frac{\gamma}{\beta}\right)\left(-\alpha+\sqrt{\alpha^{2}-\beta}\right)-v}{2 \sqrt{\alpha^{2}-\beta}},
\end{gathered}
$$

so the unique solution of the IVP (A1) has the representation as in (A2).

If $\Delta<0$ then the roots $\lambda_{1}$ and $\lambda_{2}$ are distinct and complex. We show that, formally, the solution of the IVP (A1) is the same as that in (A2). Indeed, although on the left-hand side of (A2) some complex numbers appear, due to the uniqueness of the solution of the IVP (A1), we only need to show that the left-hand side of (A2) is actually real. To see this, we define the real number $\omega$ by

$$
\sqrt{\alpha^{2}-\beta}=i \omega
$$

Then, the formula from (A2) becomes 


$$
\begin{aligned}
& y(t)=\frac{\gamma}{\beta}+\frac{\left(u-\frac{\gamma}{\beta}\right)(\alpha+i \omega)+v}{2 i \omega} e^{-\alpha t}(\cos \omega t+i \sin \omega t) \\
& +\frac{\left(u-\frac{\gamma}{\beta}\right)(-\alpha+i \omega)-v}{2 i \omega} e^{-\alpha t}(\cos \omega t-i \sin \omega t) \\
& =\frac{\gamma}{\beta}+\frac{\left(u-\frac{\gamma}{\beta}\right) \alpha+v}{2 i \omega} e^{-\alpha t} \cos \omega t+\frac{u-\frac{\gamma}{\beta}}{2} e^{-\alpha t} \cos \omega t \\
& +\frac{\left(u-\frac{\gamma}{\beta}\right) \alpha+v}{2 \omega} e^{-\alpha t} \sin \omega t+i \frac{u-\frac{\gamma}{\beta}}{2} e^{-\alpha t} \sin \omega t \\
& -\frac{\left(u-\frac{\gamma}{\beta}\right) \alpha+v}{2 i \omega} e^{-\alpha t} \cos \omega t+\frac{u-\frac{\gamma}{\beta}}{2} e^{-\alpha t} \cos \omega t \\
& +\frac{\left(u-\frac{\gamma}{\beta}\right) \alpha+v}{2 \omega} e^{-\alpha t} \sin \omega t-i \frac{u-\frac{\gamma}{\beta}}{2} e^{-\alpha t} \sin \omega t .
\end{aligned}
$$

Hence, observing that the second term cancels with the sixth term and the fifth term cancels with the ninth term, we get the representation of the solution $y$ as in (A4).

Finally, let $\Delta=\alpha^{2}-\beta=0$. In this case, the characteristic polynomial of the homogeneous second-order ODE associated to (20) has only one root $\lambda=-\alpha$ of multiplicity 2 , so we have to find the solution of the IVP (A1) in a direct fashion. Thus, the general solution of the ODE (A1) is

$$
y(t)=C_{1} \exp (-\alpha t)+C_{2} t \exp (-\alpha t)+\frac{\gamma}{\beta},
$$

and we have to determine the constants $C_{1}$ and $C_{2}$ such that initial conditions $y(O)=u$ and $y^{\prime}(0)=v$ are fulfilled. Thus, from $y(O)=u$ we get

$$
C_{1}+\frac{\gamma}{\beta}=u
$$

and since

$$
y^{\prime}(t)=-C_{1} \alpha e^{-\alpha t}+C_{2}\left(e^{-\alpha t}-t \alpha e^{-\alpha t}\right),
$$

from $y^{\prime}(0)=v$ we have

$$
-C_{1} \alpha+C_{2}=v
$$

Thus, from (A8) and (A9) we get 


$$
C_{1}=u-\frac{\gamma}{\beta}, \quad C_{2}=v-\left(\frac{\gamma}{\beta}-u\right) \alpha
$$

hence

$$
\begin{aligned}
y(t)= & \frac{\gamma}{\beta}+\left(u-\frac{\gamma}{\beta}\right) e^{-\alpha t}+\left(v-\left(\frac{\gamma}{\beta}-u\right) \alpha\right) t e^{-\alpha t} \\
& =\frac{\gamma}{\beta}+e^{-\alpha t}\left[\left(u-\frac{\gamma}{\beta}\right)+\left(v-\left(\frac{\gamma}{\beta}-u\right) \alpha\right) t\right]
\end{aligned}
$$

and then, since $\beta=\alpha^{2}$, we get (A3). QED.

The fact that the solution $y$ of the IVP (A1) has different analytic expressions with respect to $\alpha^{2}-\beta$ non-null and null can cause difficulties. In the next proposition, we show that these difficulties are not essential, more precisely, the representation (A3) is the uniform limit of the representation (A2).

Proposition 5.2. With notation as in Proposition 5.1, the solution $\mathrm{y}$ is uniformly continuous on compact subsets with respect to the parameters $\alpha, \beta>0, \gamma, u, v \in \mathbb{R}$ and $t \in \mathbb{R}$.

Proof. Using the power series expansions

$$
\exp \left(\sqrt{\alpha^{2}-\beta} t\right)=\sum_{n=0}^{\infty} \frac{\left(\alpha^{2}-\beta\right)^{n / 2} t^{n}}{n !}, \quad \exp \left(-\sqrt{\alpha^{2}-\beta} t\right)=\sum_{n=0}^{\infty} \frac{(-1)^{n}\left(\alpha^{2}-\beta\right)^{n / 2} t^{n}}{n !}
$$

in (A2) for $\alpha^{2}-\beta \neq 0$ we get

$$
y(t)=\frac{\gamma}{\beta}+e^{-\alpha t}\left[u-\frac{\gamma}{\beta}+\sum_{n=1}^{\infty} \frac{a_{n}}{n !}\left(\alpha^{2}-\beta\right)^{\frac{n-1}{2}} t^{n}\right]
$$

where, for all integers $\mathrm{n} \geq 0$,

$$
a_{n}= \begin{cases}\left(u-\frac{\gamma}{\beta}\right) \sqrt{\alpha^{2}-\beta}, & n=2 k, k \geq 0, \\ \alpha\left(u-\frac{\gamma}{\beta}\right)+v, & n=2 k-1, k \geq 1 .\end{cases}
$$

Consequently, the function $\mathrm{y}$ has continuous extension for all $\alpha, \beta>0$ and $\gamma, u, v, t \in \mathbb{R}$. Since the power series are uniformly continuous on any compact subsets, we can interchange the limit when $\alpha^{2}-\beta \rightarrow 0$ with the series, uniformly with respect to all variables, and get

$$
\lim _{\alpha^{2}-\beta \rightarrow 0} y(t)=\frac{\gamma}{\alpha^{2}}+e^{-\alpha t}\left[\left(u-\frac{\gamma}{\alpha^{2}}\right)+\left(v-\left(\frac{\gamma}{\alpha^{2}}-u\right) \alpha\right)\right] t
$$

hence this extension coincides with the definition of y provided in Proposition 5.1. QED. 